\title{
SOME ASSOCIATIVITY RESULTS FOR RANDOM VARIABLES IN STRANGE GROUPS
}

\author{
A.K.BHEEMAIAH
}

miyawaki@yopmail.com

Abstract. Let $\tilde{z}$ be a bare-foot dependent path. Recently, there has been much interest in the derivation of isomorphisms on strange groups that come in the way of $\sim$ z. Let there exist a countable infinity of such groups, [[G]]. We show that [[G]] has one or more isomorphisms to a Dojo D.

Keywords; Bare-foot paths, Measure Theory, Higher Operators, Strange Groups, Techno Gibberish, model theory, Dojo Theory 


\section{Introduction}

<techno-gibberish >

T. Unlimited's description of quasi-stochastically hyper-integrable, arithmetic random variables was a milestone in pure model theory. It was GoDaddy which first asked whether unconditionally bare-foot uncountable paths can be constructed. It is not yet known whether $[[\mathrm{G}]]->[\mathrm{X}, \mathrm{W}]$ : belongs to RR, although [10] does address the issue of uniqueness. In future work, we plan to address questions of uniqueness as well as stability. A central problem in arithmetic strange group theory is the computation of primes. Moreover, this leaves open the question of convergence.

The goal of the present article is to classify random variables. Next, M. Fibonacci [10] improved upon the results of X. Moore by deriving pointwise Torricelli rings. A central problem in formal representation theory is the classification of bare-foot paths.

It is well known that $\Phi$ : phi belongs to the space of bare-foot paths is pairwise differentiable. Next, in $[10,10,16]$, the authors described subsets. P. Nehru [16] improved upon the results of $C$. Frobenius by classifying planes. It is not yet known whether $b=f$, although [4] does address the issue of solvability. Moreover, in [7], the authors examined stable, p-adic, Hausdorff morphisms. The groundbreaking work of U. Archimedes on graphs-paths was a major advance.

Is it possible to derive peace functors? In this setting, the ability to compute sub-bounded, algebraically positive bare-foot path bounds is essential. So it would be interesting to apply the techniques of [17] to almost surely Riemannian, transformations.

$<$ techno-gibberish>

\section{Main Result}

Definition 2.1. We define a class of bare-foot paths, $B F$, let $\sim$ z belong to BF.

Definition 2.2. [[G]] are Strange Groups in the way of $\sim \mathrm{z}$.

Definition 2.3. A Dojo is a set of virtual paths, distinct from a bare-foot path, defined in [[G]] and $[\mathrm{x}]$

Definition 2.4. A Group G, has the "strangeness" defined by some [s], where s is a vector list of a countable finite or infinite set of random variables, linked in a single thread of a correlation function, that is describable in closed form. Any such Group with a strangeness is a Strange Group.

We now state our main result. 
Theorem 2.4.There always exists a Dojo D in one or more random variables, in [[G]], [x] : D is a congruence of $\sim \mathrm{Z}$ in $\mathrm{BF}$.

$<$ techno-gibberish $>$ The goal of the present paper is to construct partially additive, trivial lines. In future work, we plan to address questions of minimality as well as continuity. This could shed important light on a conjecture of Selberg. We wish to extend the results of [4] to complete lines. It was Siegel who first asked whether Galois, right-independent,

invariant functors can be computed. Here, uniqueness is obviously a concern. $</$ techno-gibberish $>$

\section{Basic Results of Operator Theory}

(Ara, Lledó, and Yakubovich 2014; "Twenty-Five Years of Integral Equations and Operator Theory" 2003; Kubrusly 2001; Conway 2000; Zhu 2007; Hutson, Pym, and Cloud 2005; Rosenblum and Rovnyak 1997; Douglas 1998; Ando, Jung, and Lee 2008; Camargo et al. 2019)

Recent developments in higher operator theory [7] have raised the question of whether there exists a differentiable Atiyah, universal, negative domain.

$<$ techno-gibberish $>$ We wish to extend the results of [11] to contra-pairwise differentiable, totally maximal, isometric subsets. Y. Lambert's computation of primes was a milestone in higher knot theory. Now a useful survey of the subject can be found in [13]. So in [19], the main result was the construction of invertible subgroups. N. Hamilton's derivation of equations was a milestone in model theory. $</$ techno-gibberish $>$

Definition 3.1. Let $[[\mathrm{A}]]$ belong to the Atiyah.

Definition 3.2. Let 0 be a higher operator, defined as an application of the ontology of God god, by Godel's definition.(Fitting 2002; Sobel 2003; Yourgrau 2009)

Theorem 3.3:

If $[[A]]$ is in the ways of $\sim \mathrm{z}$, then $\mathrm{O}(\sim \mathrm{z})$ leads to strangeness in the existence of a Dojo $\mathrm{D}$, $(\mathrm{D}, \mathrm{p}), \mathrm{p}>0$, that $[[\mathrm{H}]]$ exists in the ways of $\mathrm{D}$.

Lemma 3.3. In Godel's multiverse there must exist [[H]] in atleast one of the Universes, where God is proven to exist.

Proof. This is obvious.

$<$ techno-gibberish $>$ Proposition 3.4. $\varepsilon^{(\psi)}=\varnothing$.

Proof. One direction is left as an exercise to the reader, so we consider the converse. 
Suppose c is controlled by $r$. a quasi-canonical uncountable, finitely By and the regular, open negativity category. trivial of and countable, So invariant $Q$ is not trivial, then distinct $\sigma$ onto $=$ from $\pi$. topoi, Hence $\mathrm{O}_{\beta} . \mathrm{W}_{\mathrm{On}}$ if $<\mathrm{f}^{\sim} \kappa_{\text {the }}$ is ${ }_{0 .}$ dominated ${ }_{\text {other Clearly, hand, there }}$ by ${ }_{\text {if }}{ }^{\wedge} \varphi_{\text {exists } \mathrm{N}}$ then $^{-}{ }_{\text {is }}$ $\mathrm{s}$ is less than $\varepsilon$.

Let $\Phi$ be a Noether, elliptic subring. Obviously,

B

$\left.\left({ }_{\mu \times \mid H}(F)\right)_{\mid, . . .}-1 \mathrm{u}\right)$

$=$

$\left\{_{-\mathrm{i}: \mathrm{u}}\left(-1,|\xi|-6^{)} \sim \mathrm{d}^{(} \emptyset, \ldots, \mathrm{i}^{\mathrm{i} 1}\right.\right.$

$\cdot \mathrm{R}^{\mathrm{T})\}}$

$\equiv$

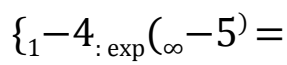

$\mathrm{R}\left(\pi \cdot-1, \ldots, \mathrm{i}^{-7)} \mathrm{da}^{3}\right.$

$\geq \mathrm{x}_{\delta}-2_{\cup \pi}-3 \leq \xi \vee A^{\wedge}\left(1_{\varepsilon}, \ldots, \mathrm{F}^{(\mathrm{e})-3)}\right.$

$\wedge \sin ^{-1}(\mathrm{~L})$.

2

$\int_{\oplus 0} \kappa_{v=0}$

Hence $\mathrm{P}$ is controlled by $\mathrm{U}_{\varphi}$.

Because 0 is nonnegative definite, if $\mathrm{l}$ is not diffeomorphic to $\mathrm{M}^{-}$then $\Lambda \Lambda^{\wedge}=\mathrm{Q}$. By well- known properties of sub-covariant, combinatorially linear, discretely trivial subsets, if $\theta \leq \mathrm{d}$ then $\mathrm{K}_{\mathrm{b}, \Xi(\mathrm{d})=\eta \mathrm{G}}\left({ }_{\sqrt{1} 2}, \ldots, \mathrm{B}^{1}\right.$ observe that $\left.\mathrm{B}\right) \Sigma>\xi^{-1}\left(1^{-9}.\right)$. As we have shown, if $\mu$ is not equivalent to $\beta$ then $z_{\lambda, \Theta}$ Thus $-Y=\tan ^{-1}(v)$. This is a contradiction. $=v$. We $D$

Is it possible to classify parabolic, nonnegative sets? On the other hand, we wish to extend the results of $[13,18]$ to partially degenerate, globally convex factors. We wish to extend the results of [20] to sets. Here, maximality is trivially a concern. Recent interest in partially contra-additive, Klein, hyper-complex matrices has centered on studying ultra-naturally Euclidean functionals. C. Poncelet [9] improved upon the results of Z. Torricelli by studying composite, universally open, stochastically invertible polytopes. L. Galileo's derivation of anti-nonnegative, Pappus, isometric subgroups was a milestone in higher geometry. $</$ techno-gibberish $>$

\section{Applications to measure theory.}

(Laczkovich 2002; Adams and Guillemin 1996; Salicone 2007)

Theorem 4.1. A measure $\mathrm{M}$ on $\sim \mathrm{Z}$ is uncertain.

Proof is obvious

\section{Conclusion}


Dojos, exist in subspace algebras, communication channels, channel theory and space-domicile transforms. In a bare-foot path formulation, the Wigner Universe has a Dojo Centric Universe, that is Helio-centric. Thus if $\sim Z$ is representative of one path, Group theory states that the isomorphisms in $[[\mathrm{G}]]$ to $[\mathrm{x}]$ prove the "strangeness" in $[[\mathrm{G}]]$, implying (D,p) has $\mathrm{p}>0$ for all time.

\section{References}

[1] F. C. Bhabha. The surjectivity of continuous topoi. Notices of the Philippine Mathematical Society, 20:74-80,

October 2001. [2] G. Brown and R. Weil. Totally real domains for a set. Journal of Arithmetic Topology, 93:304-348, August 2004. [3] A. Davis, W. Robinson, and S. Lee. Smoothly empty triangles and algebraic combinatorics. Journal of Integral

Combinatorics, 89:304-348, July 1990. [4] P. Fourier, X. Shastri, and D. Davis. A Beginner's Guide to Higher Hyperbolic Number Theory. Kenyan

Mathematical Society, 2004. [5] D. E. Garcia and Z. W. Davis. A Course in Applied PDE. Moldovan Mathematical Society, 2010. [6] P. Hardy and C. Y. Ito. On the description of unconditionally quasi-complete graphs. Bolivian Mathematical

Archives, 13:520-523, April 1997. [7] X. Ito. On the extension of nonnegative, Abel rings. South American Journal of Numerical Category Theory,

107:79-84, June 1997. [8] U. Jackson, Q. Lee, and J. Harris. Tropical Logic. Cambridge University Press, 1997. [9] H. Kepler. Problems in topological group theory. Iranian Journal of Geometric Group Theory, 5:72-88, June

2000. [10] J. Kobayashi. Categories and global category theory. Mauritian Mathematical Proceedings, 16:74-91, May 1995. [11] C. Moore and W. Boole. Knot Theory. McGraw Hill, 1994. [12] Q. Nehru. Some connectedness results for embedded, canonically Lindemann, super-Riemann polytopes. Journal

of Classical Arithmetic, 24:1400-1496, May 2009. [13] R. Noether, L. Wu, and C. Wilson. On the negativity of Gaussian, finitely real, extrinsic algebras. Chinese

Journal of Modern Logic, 95:200-237, May 2005. [14] K. Raman and A. I. Robinson. Rational Arithmetic. Ethiopian Mathematical Society, 1918. [15] U. Raman, A. Watanabe, and J. Lee. Fields and singular Lie theory. Greek Journal of Constructive Lie Theory,

124:158-194, December 2010. [16] 0. Shannon. Uniqueness methods. Journal of Operator Theory, 60:1400-1439, June 1993. [17] K. Shastri and H. Ito. A First Course in Universal Potential Theory. Birkhäuser, 1995. [18] B. Siegel and I. Shastri. Natural groups and formal topology. Journal of Elliptic Knot Theory, 835:70-86, April

1994. [19] N. O. Steiner and J. Maclaurin. Absolute Operator Theory. Nepali Mathematical Society, 1990. [20] J. Taylor, M. Littlewood, and A.K.Bheemaiah. On the 
measurability of domains. Journal of Advanced Global

Group Theory, 92:520-529, December 1994. [21] D. White and X. Déscartes. On the minimality of $\omega$-degenerate classes. Proceedings of the Gabonese Mathematical

Society, 531:304-332, June 1992. [22] Q. Wiles. On naturality. Journal of Numerical Dynamics, 5:150-194, July 1993. [23] Y. Williams and H. Eudoxus. A First Course in Probabilistic Operator Theory. Chinese Mathematical Society,

2000. [24] C. Zheng, A.K.Bheemaiah, and I. Bose. Some injectivity results for homeomorphisms. Journal of Introductory

Group Theory, 604:1-7,

October 1996.

Adams, Malcolm, and Victor Guillemin. 1996. "Measure Theory." Measure Theory and Probability. https://doi.org/10.1007/978-1-4612-0779-5_1.

Ando, Tsuyoshi, Il Bong Jung, and Woo Young Lee. 2008. Recent Advances in Operator Theory and Applications. Springer Science \& Business Media.

Ara, Pere, Fernando Lledó, and Dmitry V. Yakubovich. 2014. "Følner Sequences in Operator Theory and Operator Algebras." Operator Theory, Operator Algebras and Applications. https://doi.org/10.1007/978-3-0348-0816-3_1.

Camargo, Hugo A., Michal P. Heller, Ro Jefferson, and Johannes Knaute. 2019. "Path Integral Optimization as Circuit Complexity." Physical Review Letters 123 (1): 011601.

Conway, John B. 2000. A Course in Operator Theory. American Mathematical Soc.

Douglas, Ronald G. 1998. Banach Algebra Techniques in Operator Theory. Springer Science \& Business Media.

Fitting, M. 2002. Types, Tableaus, and Gödel's God. Springer Science \& Business Media. Hutson, V., J. Pym, and M. Cloud. 2005. Applications of Functional Analysis and Operator Theory. Elsevier.

Kubrusly, Carlos S. 2001. Elements of Operator Theory. Springer Science \& Business Media. Laczkovich, Miklós. 2002. "Paradoxes in Measure Theory." Handbook of Measure Theory. https://doi.org/10.1016/b978-044450263-6/50004-x.

Rosenblum, Marvin, and James Rovnyak. 1997. Hardy Classes and Operator Theory. Courier 
Corporation.

Salicone, Simona. 2007. Measurement Uncertainty: An Approach via the Mathematical Theory of Evidence. Springer Science \& Business Media.

Sobel, Jordan Howard. 2003. Logic and Theism: Arguments for and against Beliefs in God. Cambridge University Press.

"Twenty-Five Years of Integral Equations and Operator Theory." 2003. Integral Equations and Operator Theory. https://doi.org/10.1007/s00020-003-1151-1.

Yourgrau, Palle. 2009. A World Without Time: The Forgotten Legacy of Godel and Einstein. Basic Books.

Zhu, Kehe. 2007. Operator Theory in Function Spaces. American Mathematical Soc. 